\title{
DIGITAL COLOR REPRODUCTION AND DOCUMENTATION OF OIL PAINTING USING IMAGE PROCESSING
}

\author{
H. R. Song, Y. H. Jo* \\ Dept. of Cultural Heritage Conservation Sciences, Kongju National University - (skv9483@naver.com, joyh@kongju.ac.kr)
}

KEY WORDS: Color Management System, Color Reproduction, Image Processing, Digital Documentation, Oil Painting

\begin{abstract}
:
Digital photography is a method easily employed to capture images. Mobile devices such as smartphones and tablets as well as dedicated cameras are used to capture images. Furthermore, as hardware continues to improve, the resolution of images in $100 \mathrm{MP}$ and $8 \mathrm{~K}$ videos has recently been increased, and the quality of images enhanced through various types of image processing software. However, because the focus of photography is to record a moment activity, recording the orginal color of subject has been pushed back from priority. Digital photographs of cultural heritage are mainly captured for aesthetic appreciation and database construction. Those images for the purpose of the latter should record the exact color and shape of the subject. However, the theoretical approach of this is difficult and relatively complex procedures are able to create images that differ from the original color. Thus, it means falling in value that culture heritage data of important for color conservation. Various attempts have been made throughout the industry to capture the original colors of the subject accurately. The color management system (CMS) is a typical method of such. In this study, digital color reproduction and record studies were conducted on representative oil paintings of modern times in Korea. Accordingly, the light source's color index and color temperature were measured. Furthermore, a color recording environment was established and digital images were acquired. Subsequently, it was able to record the original color through CMS-based image processing. It is believed that this study will enable one to understand the production techniques of works through the color record of oil paintings and help one to monitor conservation conditions such as discoloration.
\end{abstract}

\section{INTRODUCION}

Digital photography is an accessible and widely used method that can record a moment activity. However, images intended for cultural heritage databases should record the exact color of the subject. Unfortunately, this is not always easy to apply digital image processes. However, the theoretical approach of this is difficult and relatively complex procedures are able to create images that differ from the original color. It means falling in value that culture heritage data of important for color conservation. Color management system (CMS) methods are typically used to accurately record the original color of the object image. In this study, digital color reproduction and documentiaion were conducted for the Korea's modern period representative oil painting. To this end, after measuring the capture environment and establishing color recording conditions, digital images were acquired. Using CMS-based image processing, it was then able to accurately reproduce the original colors of the painting. It is believed that this study will enable to understand the production techniques of works through the color record of oil paintings and help one to monitor conservation conditions such as discoloration.

\section{MATERIALS AND METHODS}

Still Life with Flowers is an oil painting by modern Korean artist, Pai, Un-soung. The first Korean to study art in Europe, Pai was active in the European art world throughout the 1920s and 1930s. At that time, he won prizes for his self-portraits and various works depicting his family members. In this study, it was applied image processing techniques to a digital photograph of Pai's colorful oil painting. The artwork was photographed with a 50 MP resolution camera and a $50 \mathrm{~mm}$ lens. Color chart were measured by using a spectrophotometer. It is then conducted camera color accuracy analysis, color correction, color space setting, and color reproduction accuracy analysis in that order.

\section{RESULTS}

\subsection{Set Reference Color}

Patch of uniform color was needed for set the reference color. So, it was used for the color chart of 140 different color patches. These patches also have different CIEL ${ }^{*} a^{*} b^{*}$ coordinates. CIEL ${ }^{*} \mathrm{a}^{*} \mathrm{~b}^{*}$ of color chart was extracted using a spectrophotometer. It was set the extracted CIE $L^{*} a^{*} b^{*}$ as the calibration reference color. Image processing was subsequently performed based on reference colors.

\subsection{Digital Image Acquisition}

It needs to use the noon sun to get accurate images. Generally, this method is difficult to apply in the field. Instead, it was acquired by using the D50 flash. The flash was located at the same distance and angle on both sides of the oil painting. It was created a capturing environment with a relative color temperature of $5,200 \mathrm{~K}$ and a color rendering index of Ra97. Due to the amount of light, the camera was set to f18, 1/120s, and iso 100. Afterward, each color chart images were obtained with or without a color chart.

\subsection{Camera Color Accuracy Analysis}

Before performing color reproduction, we determined whether the camera generally outputs the correct color. The reference color and color difference were compared using the color chart imag. This resulted in a maximum difference of 32.23 and a minimum of 3.14 for 140 patches, with an average color difference of $\Delta \mathrm{E} 17.69\left(\mathrm{CIEL}^{*} \mathrm{a}^{*} \mathrm{~b}^{*}\right)$. Therefore, accurate color 
reproduction was not possible, and color reproduction using image processing was applied.

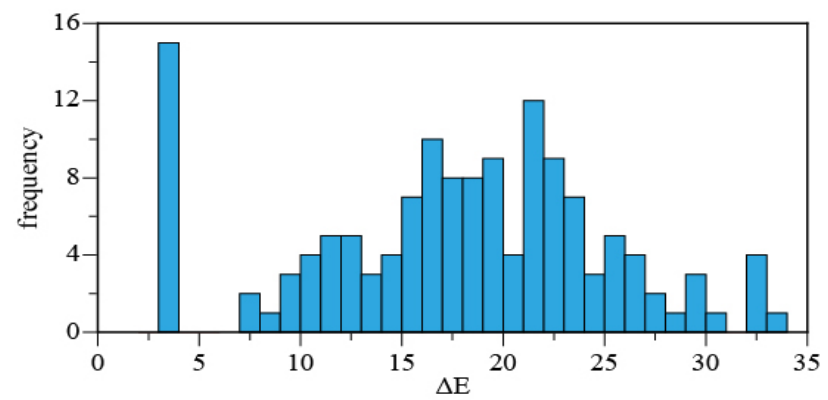

Figure 1. Camera color $\Delta \mathrm{E}$ analysis result.

\subsection{Color Correction}

As shown by the previous results, the color of general camera is not suitable for original color documentation and reproduction. Therefore, the digital color reproduction process was carried out based on the reference color. First, monitor calibration was performed that the color display remains on the consistent conditions. Afterwards, exposure, white balance, luminosity, and chromaticity were corrected to the reference color using Raw data. As a result, color change occurred visually. It then performed a distortion correction between the lens and the image sensor to calibrate the physical figures of the image.

\subsection{Establishment of Color Space}

Each digital color space has its own color area, and depending on the input/output device, RGB and CMYK are printed in various colors, even if they show the same number of colors. To solve this problem, the International Color Association (ICC) has recommended consistent color representation through color space settings. In this study, color space setting was performed using color chart images that were calibrated. At this time, it created a color space that meets ICC standards. As a result, it was possible to apply a larger color space than the space used by ordinary cameras.

\subsection{Color Reproducion Accuracy Analysis}

Color chart images that were applied with color space were compared to reference colors. This results in an average $\Delta \mathrm{E}$ $2.07\left(\right.$ CIEL $^{*} \mathrm{a}^{*} \mathrm{~b}^{*}$ ) within a color difference range of up to 7.3 to a minimum of 0.37 . As a result of color reproduction, it was able to output about 9 times more accurate colors than camera color accuracy. Previous results show that the color reproduction process is more accurate, so it synchronized the color space with the correction values it was performed so far on images that did not contain color charts. As a result, an image close to the original color of oil painting was built. In particular, the overall bright image was built close to the original color, and the chroma of various colors, such as red, blue, and green, was increased.

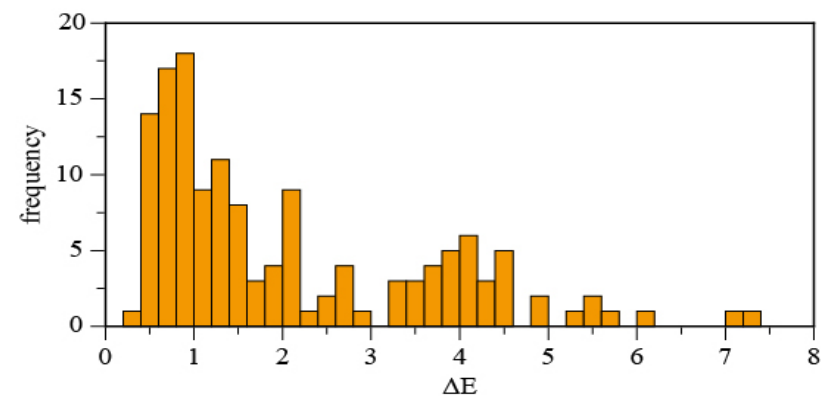

Figure 2. Color reproduction $\Delta \mathrm{E}$ analysis result.

\section{DISCUSSION}

The color of the camera was $\Delta \mathrm{E} 17.69$ and the color reproduction was $\Delta \mathrm{E} 2.07$. This is represented by the NBS color difference recognition criterion, which indicates that it has changed from "Change to another color" to "Perceivable change". Therefore, it is shown that color reproduction has been accurately completed in terms of color difference and human recognition criteria. In this study, image processing such as exposure, white balance, brightness, and chromaticity was performed, and color reproduction could be completed through color space setting of the color correction completed images. This processing was to record the exact color of the oil painting. These results could be used as a criterion for monitoring of conservation status because it was recorded close to the original color of oil painting. It is also thought that this result can be used in various ways, such as discoloration and manufacturing techniques analysis.

\begin{tabular}{|c|c|}
\hline$\Delta \mathrm{E}$ & Critical remark of color difference \\
\hline $0.0 \sim 0.5$ & Extremely slight change \\
$0.5 \sim 1.5$ & Slight change \\
$1.5 \sim 3.0$ & Perceivable change \\
$3.0 \sim 6.0$ & Marked change \\
$6.0 \sim 12.0$ & Extremely marked change \\
$12.0 \sim$ & Change to another color \\
\hline
\end{tabular}

Table 1. National Bureau of Standards NBS system of expressing color differences.
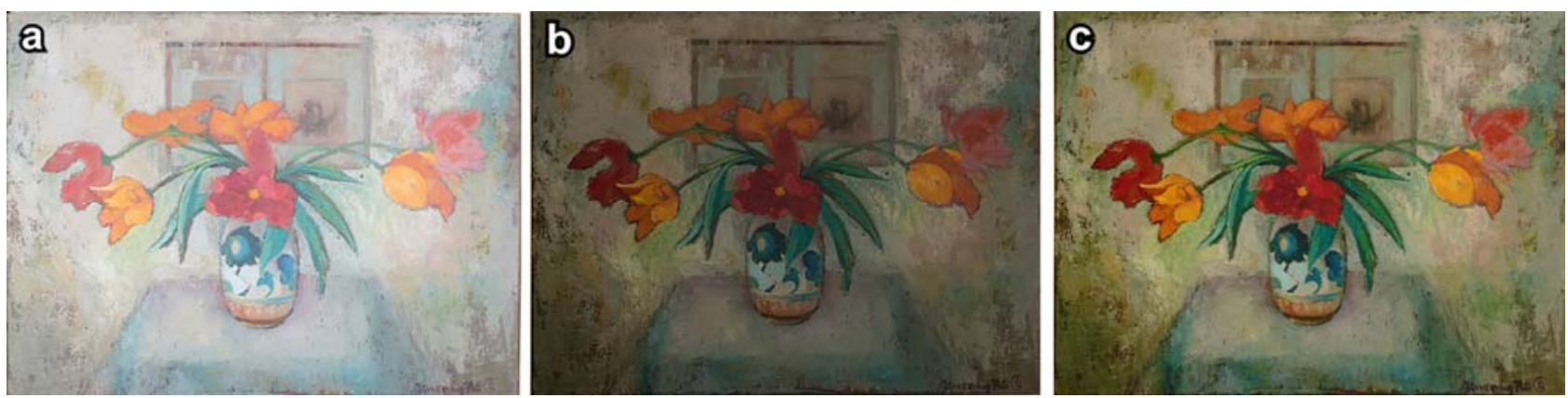

Figure 3. Image comparison according to color reproduction processing. (a) Camera profile image. (b) Color correction image. (c) Color reproduction result. 


\section{CONCLUSION}

In this study, color reproduction was completed for Still Life with Flowers. This oil painting was suitable for research because it contained a variety of colors. In this study, it is largely divided into reference color setting, image acquisition, camera color accuracy analysis, color correction, and color space setting. In particular, image processing was carried out in the order of exposure, white balance, brightness, chromaticity, lens distortion correction, and color space setting. The color of the camera was found to be a difference of $\Delta \mathrm{E} 17.69$, and the color reproduction color was 9 times more accurate $\Delta \mathrm{E} 2.07$. Therefore, exact color of the oil painting was documented. It is thought that oil painting can be used as a basis for monitoring of conservation status because it is recorded close to the original color. Also, it is believed that these results can be utilized in a variety of ways, such as analyzing manufacturing techniques or monitoring discoloration.

\section{References}

Abhay, S., 2018. Understanding color management. WILEY

Berns, R.S., 2000. Billmeyer and Saltzman's principles of color technology. WILEY.

CIE, 1932. Commission internationale de l'Éclairage proceedin gs, Cambridge University Press.

Erik, R., Michael, S., Peter, S., James, F., 2002. Photographic tone reproduction for digital images. Siggraph, 29, 267-276.

Fabrizio, I, A., Marco, G., Simone, B., 2017. Color definition of open-air Architectural heritage and Archaeology artworks with the aim of conservation. Digital Applications in Archaeology and Cultural Heritage, 7, 10-31.

Frank, H. M., 1996. Color environment, and human response. WILEY.

Kaida, X., Fareadon, Z., Julian, M, Y., 2012. Colour management system for displaying microscope images. Displays, 33, 214-220.

Kaida, X., Fareadon, Z., Richard, V.N., Julian, M.Y., 2013. Color reproduction for advanced manufacture of soft tissue prostheses. Journal of Dentistry, 41, 15-23.

Lee, E.S., Lee, S.H., Jang, K.I., Sohng, K.I., 2005. Adaptive colorimetric characterization of camera for the variation of white balance. Electron, 88, 2289-2302.

Kirillova, N, P., Zhang, Y., Hartemink, A, E., Zhulidova, D, A., Artemyeva, Z, S., Khomyakov, D, M., 2021. Calibration methods for measuring the color of moist soils with digital cameras. Catena, 202, 1-11.

Susan, W., 2006. Practical color management, Optics \& Laser Technology. 38, 399-404.

Tom, P.A., 2014. Color management \& quality output, working with color from camera to display to print. Focal Press 